Original Article

\title{
THE ROLE OF A SPECIALIST PHARMACIST IN THE MANAGEMENT OF ADALIMUMAB IN PATIENTS WITH INFLAMMATORY BOWEL DISEASE
}

\author{
JOHANNES HEGNER1, JANKI PATEL ${ }^{1}$, STEVEN FONG1, SIMON JEFFS ${ }^{2}$
}
${ }^{1}$ Department of Gastroenterology, East Sussex NHS Healthcare Trust, ${ }^{2}$ Department of Pharmacy and Biomolecular Sciences, University of Brighton
Email: s.jeffs@brighton.ac.uk

Received: 17 Jun 2021, Revised and Accepted: 03 Sep 2021

\begin{abstract}
Objective: The management of inflammatory bowel disease (IBD) patients on complex medications such as biologic disease-modifying antirheumatic drugs (DMARD) requires close supervision. At East Sussex NHS Healthcare Trust (ESHT), the multi-disciplinary team (MDT) already looking after these patients could benefit from the additional knowledge and support from a specialist pharmacist (SP).

Methods: To assess if the MDT could benefit from an SP, all IBD patients on the DMARD adalimumab were identified. The patient records were screened for patient demographic data, clinical assessment and investigations, treatment, and follow-up clinics.

Results: 162 patients at ESHT were identified as being on adalimumab treatment for either Crohn's Disease (77\%) or Ulcerative Colitis (23\%). Disease activity scores, a clinical measure of IBD severity, were infrequently recorded (1\%) on patient letters. Evidence of a biologic screen, a series of investigations to ensure safety in a biologic treatment, was only evident in one-third of patients. Clinic review of patients recently started on adalimumab and annual review of stable patients occurred $43 \%$ and $26 \%$ respectively.

Conclusion: The results indicate that there is a need for an additional member to support the IBD MDT in managing this cohort of patients. An SP is uniquely positioned to fill this gap. They have extensive knowledge in drug indication, therapeutic drug monitoring, and side-effect profiles. Similar
\end{abstract} studies have been identified that support SP in this role.

Keywords: Pharmacist, Medication, Inflammatory bowel disease

(C) 2021 The Authors. Published by Innovare Academic Sciences Pvt Ltd. This is an open-access article under the CC BY license (https://creativecommons.org/licenses/by/4.0/) DOI: https://dx.doi.org/10.22159/ijpps.2021v13i11.42451. Journal homepage: https://innovareacademics.in/journals/index.php/ijpps.

\section{INTRODUCTION}

Specialist pharmacists (SP) have increasingly been playing a role in the treatment of conditions that require complex pharmacological management [1]. The role of the pharmacist has been expanding from conventional duties to becoming more independent, allowing them to prescribe for patients within a defined scope of practice [25]. These additional duties to monitor patients and prescribe for them can reduce the workload on other healthcare professionals. This is reflected in the study by Walter et al,, which found that employing specialist clinical cancer pharmacists reduced clinic visits and reduced workload on the doctor [3]. The role of the SP in managing inflammatory bowel disease (IBD) has not been widely assessed to date. The management of IBD could lend itself well to the role of an SP considering the complex IBD drug management comprehensive monitoring for side effects, and following up on therapeutic drug monitoring (TDM). Additionally, patient education is essential towards long-term compliance and management. IBD is a condition frequently seen in gastroenterology, affecting more than 400,000 people in the UK alone [6]. It can be divided into two distinct clinical conditions-Crohn's Disease (CD) and Ulcerative Colitis (UC) [7]. CD is a chronic inflammatory condition having patchy regions of inflammation. These can be found throughout the alimentary tract but are most commonly found in the ileocecal region [7]. The inflammation is transmural, thereby affecting all the layers. In UC, the inflammation is usually contained to the rectum and proximal colon [7]. The inflammation and ulceration are contained in the colonic mucosa. The medical treatment of both conditions revolves around the use of anti-inflammatories and immunosuppressants (e. g. steroids), and disease-modifying antirheumatic drugs (DMARD)[8]. First-line medications consist of corticosteroids, aminosalicylates, and azathioprine whilst anti-tissue necrosis factor-alpha (TNF-a) agents such as adalimumab or infliximab are reserved for more moderate-severe cases $[9,10]$ These medications may require careful and regular monitoring by specialist teams in secondary care. Patients have to be assessed to ensure therapeutic drug levels, drug metabolism, immunogenicity, and side effects [11]. One of the anti-TNF-a inhibitors prescribed for patients with IBD is the monoclonal antibody adalimumab. Its mechanism of action is by inhibiting TNF-a by binding to soluble TNF-a and preventing its interaction with cellular TNF-a receptors [12]. Adalimumab is a biologic DMARD (bDMARD) and is indicated in adult patients with moderate to severe CD or UC who have had an inadequate response to conventional therapy. The inadequate response is often clinically assessed, with bowel motions per day being an important component [11]. The IBD patients on adalimumab requiring highly individualized, frequent, and complex care calls for and provides an opportunity for SPs to assist the healthcare team in monitoring and treating these patients. This paper aims to establish the current management of patients on adalimumab and to assess the need for an SP in managing patients on adalimumab at East Sussex Healthcare NHS Trust (ESHT).

\section{MATERIALS AND METHODS}

Blueteq ${ }^{\circledR}$ is an electronic platform used to monitor compliance with national guidelines[13]. At ESHT the Blueteq ${ }^{\circledR}$ database was used to identify all patients on active adalimumab treatment in November 2018. This was verified using the pharmacy dispensing record system. Patients were excluded if they could not be verified using the pharmacy dispensing record system. Once all patients on active adalimumab had been established, data was collected by using the pharmacy dispensing system and the clinical database, which provides access to patient details, laboratory results, and clinical letters. All patients were then reviewed by a virtual multidisciplinary team (MDT) meeting, which included an SP and a Consultant Gastroenterologist. The patient's management was reviewed and the potential for further assessment or change in management was evaluated. These outcomes were all recorded in an excel spreadsheet. Any actions or follow-ups required for existing 
patients that were reviewed as part of this research were fed back to the IBD specialist nurses.

Patient documents were screened for baseline patient demographics, clinical assessments and investigations, treatment, and follow-up. Clinical assessment of patients was quantified through clinical scoring systems, using either the Harvey Bradshaw Index (HBI) for CD or the Truelove and Witts score for UC $[14,15]$. Basic investigations included fecal calprotectin and patient weight. A low BMI secondary to malnutrition is common in patients with undiagnosed IBD or those who are relapsing. Fecal calprotectin is an established marker to distinguish between IBD and irritable bowel syndrome $[11,16]$.

\section{RESULTS}

\section{Patient demographics and characteristics}

The study identified 162 patients at ESHT through the Blueteq ${ }^{\circledR}$ system, which were labelled as receiving adalimumab (table 1). The average age of the patient cohort was 45 , with a standard deviation of $17 \mathrm{y}$. The majority of patients on adalimumab were being treated for CD (77\%), whilst the rest were treated for UC (23\%).
Table 1: Patient demographics and characteristics

\begin{tabular}{ll}
\hline Parameter & \\
\hline Number of Patients & 162 \\
Age (years) & $46 \pm 17$ \\
Male/Female Ratio & $69 / 93$ \\
Crohn's Disease & 125 \\
Ulcerative Colitis & 37 \\
\hline
\end{tabular}

\section{Initial assessment and investigations}

Initial assessment and investigations are listed in table 2. Disease activity was infrequently recorded in the patient letter (1\%). A review of the Blueteq ${ }^{\circledR}$ system showed that disease activity scores were documented in $77 \%$ of the cases. This can largely be explained as it is a mandatory requirement most of the time on the Blueteq ${ }^{\circledR}$ system. A minority of patients $(21 \%)$ were assessed using fecal calprotectin. The majority of patients $(76 \%)$ were investigated with further interventions. Of those documented (table 3) the most common interventions were colonoscopy, magnetic resonance imaging scans, and sigmoidoscopies.

Table 2: Initial assessment and investigations

\begin{tabular}{|c|c|c|c|}
\hline Parameters & Yes & No & Not available \\
\hline Disease activity scores on patient letter & $2(1 \%)$ & $158(98 \%)$ & $1(2 \%)$ \\
\hline Disease activity scores on Blueteq ${ }^{\circledR}$ & $124(77 \%)$ & $16(10 \%)$ & $22(13 \%)$ \\
\hline Interventions carried out & $123(76 \%)$ & $37(23 \%)$ & $2(1 \%)$ \\
\hline Pre-Biologic screen & $54(33 \%)$ & $107(66 \%)$ & $1(1 \%)$ \\
\hline Fecal Calprotectin & $34(21 \%)$ & $127(78 \%)$ & $1(1 \%)$ \\
\hline Weight documented & $9(5 \%)$ & $152(94 \%)$ & $1(1 \%)$ \\
\hline
\end{tabular}

Table 3: Further investigations to assess IBD

\begin{tabular}{ll}
\hline Colonoscopy & 47 \\
MRI & 45 \\
Sigmoidoscopy & 29 \\
Not Available & 38 \\
\hline
\end{tabular}

Table 3: Illustrates the number of investigations performed to assess the disease severity of patients with IBD before starting adalimumab treatment. Data represents the total amount; patients could have multiple and repeat investigations.

\section{Pre-biologic screening}

Before starting a patient on a biologic such as adalimumab, the British Society of Gastroenterology (BSG) guidance recommends carrying out a pre-biologic screen [11]. This is to ensure that there are no absolute contraindications to commencing treatment. At ESHT a pre-biologic screen consists of a standard blood test, a viral screen, T-spot test, a chest $\mathrm{x}$-ray, and a pregnancy test if relevant. Clinic letters only recorded that a pre-biologic screen was conducted one-third of the time (33\%). Monitoring weight trends is important in patients with IBD; however, it was rarely (5\%) documented in clinic letters.

Treatment and management of patients with IBD on adalimumab

A total of 162 patients were identified by Blueteq ${ }^{\circledR}$ as being on active adalimumab treatment, however, 25 patients $(15 \%)$ were not actively receiving the medication. Further analysis showed that some patients had developed new contraindications to the medications whilst others did not engage with the healthcare system. A large proportion (72\%) of clinic records did not indicate that patients were involved or given a choice about starting biologic treatment (table 4). The Hackett report emphasizes that patients should be involved in treatment decisions and routes of administration [17]. It found this to be beneficial for patient compliance and treatment. The treatment regime for $\mathrm{CD}$ and UC may be escalated to a more frequent dosing regimen (once every $2 \mathrm{w}$ to weekly). Data collected shows that $23 \%$ of CD patients and $35 \%$ of UC patients had their treatment escalated (table 4). After a decision to escalate treatment frequency has been taken, it is best practice to assess if the patient has developed any antibodies to the treatment and to elicit drug trough levels [11]. This was identified on clinic records as only occurring $21 \%$ of the time (table 4 ).

Biologic treatment with adalimumab should be discontinued once a prolonged corticosteroid-free remission has been observed [11]. In total, only $4 \%$ of cases showed clear documentation about duration and treatment cessation. A comparison between the treatment dose of adalimumab the patient actually receives and that recorded in the Blueteq ${ }^{\circledR}$ system is difficult to evaluate, as $98 \%$ of patients in the Blueteq ${ }^{\circledR}$ do not have their dosing frequency recorded. The Blueteq ${ }^{\circledR}$ system does not make this a mandatory requirement. This contributes to the low rate $(2 \%)$ of concurrence of the recorded dose in the Blueteq ${ }^{\circledR}$ system compared to clinic notes.

Table 4: Treatment and management of patients with IBD on adalimumab

\begin{tabular}{|c|c|c|c|c|}
\hline \multicolumn{2}{|l|}{ Parameter } & Yes & No & Not Available \\
\hline \multicolumn{2}{|l|}{ Receiving Treatment } & $136(84 \%)$ & $25(15 \%)$ & $1(1 \%)$ \\
\hline \multirow[t]{2}{*}{ Escalation by Indication } & Crohn's Disease & $29(23 \%)$ & $96(77 \%)$ & $0(0 \%)$ \\
\hline & Ulcerative Colitis & $13(35 \%)$ & $24(65 \%)$ & $0(0 \%)$ \\
\hline \multicolumn{2}{|c|}{ Levels taken on treatment escalation } & $9(21 \%)$ & $32(76 \%)$ & $1(2 \%)$ \\
\hline \multicolumn{2}{|c|}{ Patient involved in decision making } & $44(27 \%)$ & $116(72 \%)$ & $2(1 \%)$ \\
\hline \multicolumn{2}{|c|}{ Documentation stopping treatment } & $6(4 \%)$ & $155(95 \%)$ & $1(1 \%)$ \\
\hline \multicolumn{2}{|c|}{ Concurrence of Blueteq and Actual Dose } & $3(2 \%)$ & $0(0 \%)$ & $159(98 \%)$ \\
\hline
\end{tabular}




\section{Follow-up of patients on adalimumab}

The 3-month review after treatment started is an important timeframe as the clinician can assess the effectiveness against the side effects. Generally accepted practice along with BSG guidance is for a review of IBD patients on biologics within a month after the loading dose has been completed, which in this scenario equates to a 3 -month review [11]. Any review occurring $28 \mathrm{~d}$ before and after 3 mo of starting treatment was included. The review showed that only $43 \%$ of patients were reviewed within this timeframe (table 5). A regular review should occur yearly after starting adalimumab treatment $[9,10]$. A large proportion of patients did not have any data available on yearly follow-up (28\%). Almost half of the patients $(46 \%)$ did not have any evidence or did not have the annual review within the required timeframe. Only $26 \%$ of patients were seen at a one-year follow-up with a 28-day leeway on either side. Patients were followed up in the clinic more frequently than required by NICE guidelines. The records showed that $73 \%$ of patients had an additional clinic scheduled. Following data collection and MDT review $77 \%$ of patients were identified as needing a review due to no recent review in a clinic, missing information, requiring follow-up investigations, or treatment alterations.

Table 5: Follow-up of patients on adalimumab

\begin{tabular}{llll}
\hline Parameter & Yes & No & Not Available \\
\hline 3-Month Review & $69(43 \%)$ & $81(50 \%)$ & $12(7 \%)$ \\
Annual Review & $42(26 \%)$ & $74(46 \%)$ & $46(28 \%)$ \\
Additional clinic & $119(73 \%)$ & $39(24 \%)$ & $4(2 \%)$ \\
Patient review after MDT & $125(77 \%)$ & $35(22 \%)$ & $2(1 \%)$ \\
\hline
\end{tabular}

\section{DISCUSSION}

The results of this study highlight that important parameters which strongly influence patient care have a low rate of documentation. This is evidenced by $1 \%$ of clinic notes recording disease activity scores or $4 \%$ of clinic letters having treatment cessation information. Having more reliable records will allow the healthcare team to establish a trend, improve continuity of care and facilitate more accurate audits. Disease activity scores in IBD can be used as a tool to identify patients with more aggressive disease that require escalation of treatment. The data collected found only $1 \%$ of patient letters to contain a disease activity scores. A review of Blueteq $₫$, a system primarily only accessible to pharmacists, indicates $77 \%$ of patients on adalimumab have disease activity scores logged. It would be of benefit for these disease activity scores to be more accessible to the wider healthcare team. This would allow for improved continuity of care and establishing a disease trend. Similarly, the weight of a patient is also rarely documented $(5 \%)$. This again would provide benefit if a trend is established. A low BMI secondary to malnutrition is common in patients with undiagnosed IBD or those who are relapsing. These patients could therefore benefit from an escalation of their treatment [11].

As discussed, a pre-biologic screen ensures patient safety when considering initiating a biologic treatment. This screen encompasses multiple tests, including blood analysis and imaging. Patient letters infrequently (33\%) mention that this has been completed. At ESHT there is no standardized format for the completion of these tests. As this is an important reoccurring routine step of treating a patient with a biologic, this would lend itself well to a checklist. This could assure all healthcare professionals involved in the biologic management that a biologic screen has been done. It would also potentially prevent duplicate testing if accurately documented. A completed checklist would be suitable for repeated audits to ensure compliance.

Having clearly defined and documented parameters for treatment cessation is important to the next clinician assessing this patient. Treatment should not be long-term as this predisposes to an increased incidence of serious infections and risk of non-melanoma skin cancer [18]. Once a patient is initiated on adalimumab, a conversation about treatment duration should be considered. Anecdotally, unclear endpoints concerning treatment duration both increase the risk of patients being reluctant to cease treatment and increase the duration of treatment. In addition, this manages patient expectations who may not realize that the focus of the treatment is to induce remission as opposed to a lifelong strategy. Prolonged treatment would also add financial pressure on the healthcare system, not only with adalimumab being a high-cost drug but also in the form of additional clinic appointments. Lastly, as different clinicians may be reviewing this patient over time, a defined endpoint would improve continuity of care.

This data suggests that important parameters are not routinely recorded in patient letters where they are easily accessible.
Improving documentation in these identified parameters can improve patient care through improved communication. This results in better continuity of care and allows the healthcare team to establish relevant trends.

The results indicated that follow-up of patients after they have been started on a biologic shows inconsistency. The optimal time frame for follow-up in patients that are on biologic treatment should occur within 2-3 mo followed by an annual review [11]. These parameters were adhered to in only $43 \%$ of cases at the 3 -month and $26 \%$ at the annual review. These reviews ensure that patients receive the maximum benefit from the treatment, assess side effects, and review the indication of the medication. A large majority $(73 \%)$ of patients are booked into additional clinics throughout the year. Even though patients were being booked into additional clinics, it was still deemed necessary to reassess the patient care $77 \%$ of the time after an MDT meeting between a Consultant Gastroenterologist and an SP.

Reviewing patients at 3-month intervals could be a factor using up resources and time. By reducing the number of appointments and scheduling reviews annually, clinicians and healthcare staff could use their time more efficiently. As highlighted by the NICE guidelines above, the management of patients who have IBD requires multiple assessments repeatedly occurring over time to ensure optimal management. An IBD SP would be able to be involved in the antiTNF-a drug protocol used in IBD. This could include pre-screening, initiating treatment, monitoring, and ongoing treatment requirements.

A limitation encountered in this study was that some patients had been on adalimumab before the introduction of the Blueteq ${ }^{\circledR}$ database in January 2016. Although these patients were identified, this limited data collection for some parameters and could contribute towards Non-Available (NA) data. Furthermore, following the introduction of Blueteq $\AA$, the existing patients were seen as legacy patients and therefore continued on treatment regardless of NICE guidelines. During this study, significant amounts of the data were extracted from clinic letters, which are primarily written to communicate with the patient and their primary healthcare provider. Therefore, important information not relevant to these parties could have been excluded. Clinicians and allied healthcare staff did document separately on written records, however, there was no access to these archived notes. Lastly, due to limitations in funding and time, it was not possible to assess the impact an SP could make on IBD biologic management.

SPs have been noted to make a significant difference in other treatments. Mikolas et al., described the deployment of an SP in a Hepatitis C Virus treatment clinic [4]. The SP treated patients with direct-acting antivirals. Their tasks included pre-treatment screening, drug selection, therapeutic drug monitoring, adherence, and follow-up. The sustained virologic response of the patients treated under a clinical pharmacist specialist using direct-acting antivirals was similar to previous literature [4]. SPs were also 
investigated by Walter et al., who evaluated whether the complex medication for lung cancer patients could be managed by SP [3]. Evaluated over $6 \mathrm{mo}$, medication adherence and patient satisfaction improved significantly. The healthcare system benefited from a significant reduction in-clinic appointments. One study investigated the impact a pharmacist could have on outpatients receiving haemodialysis. The authors identified this group as high-risk for drug-related problems due to multiple co-morbidities and polypharmacy. The study found that having a pharmacist review patient's medications reduced ineffective and suboptimal drug therapy [19].

A study conducted by Brighton and Sussex University Hospitals (BSUH) trust, imbedded an SP into the multi-disciplinary team [20]. The SP provided an independent prescribing, TDM, and biologic infusion clinic service. Analysis following this implementation found significant cost savings, decreased clinic slots for the gastroenterology doctors and nurse specialists, and enhanced patient safety through improved TDM and standardization in patient management.

\section{CONCLUSION}

Conditions that require complex care, a multi-disciplinary approach, regular TDM, and constant re-evaluation can benefit from the supervision of a dedicated SP. The data presented in this paper along with the supporting literature, makes for an argument that the IBD management at ESHT could greatly benefit from a dedicated SP. Our observations along with the publications by Mikolas et al., Walter et $a l$, and the team at BSUH, support SP covering part of the patient care, doing this safely, and decreasing workload on other healthcare members. Our data illustrated that $73 \%$ of patients are booked into additional clinics, whilst a majority of patients are not assessed at the correct intervals. The Royal Pharmaceutical society recognizes the pharmacist's role as an independent prescriber and states that this will help "drive quality improvement in the delivery of care". Pharmacist independent prescribers will "lead to more effective models of care for" all of our patients [5]. To strengthen the claim that SP adds significant value, a cohort study investigating the impact of an SP on patient care of adalimumab for IBD at ESHT would be required.

\section{FUNDING}

Nil

\section{AUTHORS CONTRIBUTIONS}

Johannes Hegner contributed towards the analysis of the obtained data, researched similar literature and writing the manuscript. Janki Patel was involved in the study design, data collection, and the analysis of obtained data. Steven Fong and Simon Jeffs contributed to the idea of the study, the scope of the study and contributed towards the analysis of the data. All authors were involved in editing the manuscript.

\section{CONFLICT OF INTERESTS}

\section{Declared none}

\section{REFERENCES}

1. Baqir W. Helping to unlock resources: the changing role of the pharmacist; 2015. Available from: https://www.health.org.uk/ blogs/helping-to-unlock-resources-the-changing-role-of-thepharmacist. [Last accessed on $24 \mathrm{Feb} 2021$ ]

2. Cross VJ, Parker JT, Law Min M-CYLL, Bourne RS. Pharmacist prescribing in critical care: an evaluation of the introduction of pharmacist prescribing in a single large UK teaching hospital. Eur J Hosp Pharm. 2018;25(e1):e2-6. doi: 10.1136/ejhpharm2017-001267, PMID 31157059.

3. Walter C, Mellor JD, Rice C, Kirsa S, Ball D, Duffy M, Herschtal A, Mileshkin L. Impact of a specialist clinical cancer pharmacist at a multidisciplinary lung cancer clinic. Asia Pac J Clin Oncol. 2016;12(3):e367-74. doi: 10.1111/ajco.12267, PMID 25227909.
4. Mikolas LA, Jacques K, Huq M, Krasner C, Mambourg SE. Utilizing clinical pharmacist specialists to manage hepatitis $C$ virus patients on direct-acting antiviral therapy. J Pharm Pract. 2019;32(6):655-63. doi: 10.1177/0897190018777345, PMID 29783912.

5. Royal Pharmaceutical Society. Pharmacist independent prescribers; 2018.

6. Pasvol T], Horsfall L, Bloom S, Segal AW, Sabin C, Field N, Rait G. Incidence and prevalence of inflammatory bowel disease in UK primary care: a population-based cohort study. BMJ Open. 2020;10(7):e036584. doi: 10.1136/bmjopen-2019-036584, PMID 32690524.

7. Sairenji T, Collins KL, Evans DV. An update on inflammatory bowel disease. Prim Care. 2017;44(4):673-92. doi: 10.1016/j.pop.2017.07.010, PMID 29132528.

8. Jeong DY, Kim S, Son MJ, Son CY, Kim JY, Kronbichler A, Lee KH, Shin JI. Induction and maintenance treatment of inflammatory bowel disease: a comprehensive review. Autoimmun Rev. 2019;18(5):439-54. doi: 10.1016/j.autrev.2019.03.002, PMID 30844556.

9. National Institute for Health and Care Excellence. Infliximab and adalimumab for the treatment of Crohn's disease; 2010. Available from: https://www.nice.org.uk/guidance/ta187/chapter/1Guidance. [Last accessed on $28 \mathrm{Feb} 2021$ ].

10. National Institute for Health and Care Excellence. Infliximab, adalimumab and golimumab for treating moderately to severely active ulcerative colitis after the failure of conventional therapy; 2015. Available from: https://www.nice.org.uk/guidance/ta329. [Last accessed on 28 Feb 2021]

11. Lamb CA, Kennedy NA, Raine T, Hendy PA, Smith PJ, Limdi JK, Hayee B, Lomer MCE, Parkes GC, Selinger C, Barrett KJ, Davies RJ, Bennett C, Gittens S, Dunlop MG, Faiz O, Fraser A, Garrick V, Johnston PD, Parkes M, Sanderson J, Terry H, IBD guidelines eDelphi consensus group, Gaya DR, Iqbal TH, Taylor SA, Smith M, Brookes M, Hansen R, Hawthorne AB. British society of gastroenterology consensus guidelines on the management of inflammatory bowel disease in adults. Gut. 2019;68(Suppl 3):s1s106. doi: 10.1136/gutjnl-2019-318484, PMID 31562236.

12. Levin AD, Wildenberg ME, van den Brink GR. Mechanism of action of anti-TNF therapy in inflammatory bowel disease. J Crohns Colitis. 2016;10(8):989-97. doi: 10.1093/eccojcc/jjw053, PMID 26896086.

13. Blueteq Ltd. Trust HCD IFR System. Available from: http://www.blueteq.com.Systems/Trust-HCD-IFRSystem.html. [Last accessed on 28 Feb 2021]

14. Truelove SC, Witts LJ. Cortisone in ulcerative colitis; final report on a therapeutic trial. Br Med J. 1955;2(4947):1041-8. doi: 10.1136/bmj.2.4947.1041, PMID 13260656.

15. Harvey RF, Bradshaw JM. A simple index of Crohn's disease activity. Lancet. 1980;1(8167):514. doi: 10.1016/s01406736(80)92767-1, PMID 6102236.

16. National Institute for Health and Care Excellence. Faecal calprotectin diagnostic tests for inflammatory diseases of the bowel. Nice; 2013. Available from: https://www.nice.org.uk/ guidance/dg11/chapter/1-Recommendations. [Last accessed on $28 \mathrm{Feb} 2021]$

17. Hackett M. Handbook for home care services in England; 2014.

18. Burmester GR, Panaccione R, Gordon KB, Mcllraith MJ, Lacerda APM. Adalimumab: long-term safety in 23458 patients from global clinical trials in rheumatoid arthritis, juvenile idiopathic arthritis, ankylosing spondylitis, psoriatic arthritis, psoriasis and Crohn's disease. Ann Rheum Dis. 2013;72(4):517-24. doi: 10.1136/annrheumdis-2011-201244, PMID 22562972.

19. Lumbantobing R, Sauriasari R, Andrajati R. Role of pharmacists in reducing drug-related problems in hemodialysis outpatients. Asian J Pharm Clin Res. 2017;10(17):108-13. doi: 10.22159/ajpcr.2017.v10s5.23110.

20. Jones A, Smith M. Embedding pharmaceutical care into the multidisciplinary team. Brighton and Sussex Hospitals NHS Trust; 2014. 